\title{
Upslope Flows in Atmosphere and Water Tank, Part I: Scaling
}

\author{
C. Reuten", D.G. Steyn, and S. E. Allen
}

\author{
Atmospheric Science Program, Department of Earth and Ocean Sciences, The University of British Columbia, \\ Vancouver, Canada
}

\begin{abstract}
Upslope flows are a crucial mechanism in the transport of air pollutants in complex terrain, both as separate flow systems and as part of other thermally driven flows. Resolving steep complex terrain in numerical models requires horizontal resolutions that are difficult to achieve. Water-tank models of upslope flows provide additional insights but require idealizations that have typically limited comparisons with atmospheric observations to order-of-magnitude estimations. This paper applies scaling to a water tank that was specifically designed to achieve quantitative similarity with field measurements at a particular site. Non-dimensional boundary-layer depths near the base of slope in atmosphere and water tank agree within the measurement uncertainties of the field observations $(20 \%)$. We show that boundary-layer depth and upslope flow velocity at any point in time are completely determined by instantaneous and integrated surface heat fluxes (from the beginning of positive heat flux to the point in time), regardless of the surface heat flux's particular path in time. While velocities in two independent tank experiments with steady and sinusoidal surface heat flux, respectively, agree reasonably well at the expected time of similarity, they disagree statistically significantly with velocities in the atmosphere. This disagreement implies a dependence on molecular quantities (viscosity, thermal diffusivity). Since different definitions of Reynolds numbers provide inconclusive values and both the appropriate velocity scale and length scale for a Reynolds number are functions of the flow itself, we derive an alternative set of governing parameters. This set provides the basis for a detailed hypothesis for the similarity violation of upslope flow velocities in atmosphere and water tank in a companion paper.
\end{abstract}

Keywords: Anabatic flows, physical scale model, scaling, similarity, upslope flows, water tank.

\section{INTRODUCTION}

Under fair weather conditions, surface heating over a slope creates a horizontal temperature gradient between points near the slope surface and points at the same height further from the slope, causing thermally driven upslope flows. These are ubiquitous in complex terrain as individual flows or as part of larger scale flows. In up-valley flows, for example, upslope flows occur over sloping valley bottoms and over the valley side walls, where they redistribute heat in the valley cross section creating along valley temperature gradients. Despite the importance of upslope flows, their quantitative properties and their role in air-pollution dispersion are not yet fully understood. Upslope flows often reduce air-pollutant concentrations in mountainous terrain by venting air pollutants out of the boundary layer into the free atmosphere [1]. Field observations at a $19^{\circ}$ slope in Minnekhada Park in the Lower Fraser Valley, British Columbia, Canada, and a close look at previous field, laboratory, numerical, and analytical studies demonstrate that this is not always the case; it seems that air pollution can be trapped or re-circulated within the boundary layer [2]. A bottom-heated water-tank with a plain and a plateau connected by a $19^{\circ}$ slope was built as an idealization of the Minnekhada Park site. Experiments with dye injected over the tank bottom served to investigate air-pollutant transport

*Address correspondence to this author at the RWDI AIR Inc., Suite 1000, $7368^{\text {th }}$ Ave. SW, Calgary, AB T2P 1H4, Canada; Tel: 1-403-232-6771; Fax.: 1-403-232-6762; Email: creuten@gmail.com in upslope flows and to measure backscatter boundary layer (BBL) depth. Upslope flow velocities were measured with neutrally buoyant particles evenly distributed in the tank. This paper attempts to compare BBL depths and upslope flow velocities in the water tank and at Minnekhada Park within the uncertainty of the field observations (about 20\%).

Scaling has been used for hundreds of years as a powerful tool in applied mathematics and engineering and is often interpreted differently by different investigators [3]. The central goal of scaling in this paper goes back to Tolman's [4] principle of similitude, which requires that two universes of different scales are exactly similar. Tolman postulated the principle "as a temporary criterion for the correctness of physical theories", hence as a screening tool. Generalizing Tolman's work, Buckingham [5] developed the formalism of dimensional analysis, which is well explained in Barenblatt [3] and which will be applied in this paper.

Tritton [6] describes two routes to determining under what conditions similar flows will occur in two different systems. The first route is based on gathering all quantities that are physically relevant for differences between the systems. The second route begins with the governing equations. In Tritton's example (p. 89 f.), the first route is faster, but for more complicated problems, Tritton points out that this method is harder to apply than the one based on the governing equations. The latter method appears less ad hoc and mathematically more rigorous, but the appropriate form of the governing equations and initial and boundary conditions may be difficult to determine. One of the 
advantages of the first method is that the non-dimensional groups are dependent only on the externally set parameters whereas the second method generates non-dimensional groups dependent on "observed" phenomena such as the velocity and the boundary layer thickness. For this reason we follow the first method.

Previous investigators achieved an order-of-magnitude agreement between atmospheric and water-tank observations of upslope flow phenomena [7, 8]. However, their formulations disagree with each other and with large-eddy resolving numerical simulations [9]. Here we ask if we can get true quantitative similarity between the atmosphere and tank. Thus, this paper builds on these earlier studies by using an experimental setup particularly designed to be tested against atmospheric observations at Minnekhada Park.

This paper is laid out as follows. The main characteristics of field site and water tank are summarized in section 2 . Section 3 describes the scaling and section 4 results of its application to upslope flows in the atmosphere and the water tank. The discussion in section 5 is followed by conclusions in section 6 .

\section{CHARACTERISTICS OF FIELD SITE AND WATER TANK}

\section{a. Field Site}

A detailed description of the field site and instrumentation at Minnekhada Park in the Lower Fraser Valley, British Columbia, Canada, can be found in [2]. The slope is fairly homogeneous over a width of roughly $3 \mathrm{~km}$; the slope angle is approximately constant at $19^{\circ}$ (Fig. 1a). The ridge height is $760 \mathrm{~m}$. Fig. (2) in [2] suggests that the terrain on the other side of the ridge is better represented by a plateau than a slope. The plain adjacent to the slope is approximately flat. Observations used in this paper were taken under mostly cloudless skies in the morning hours of 25-26 July 2001, before the onset of sea-breeze and upvalley flows. Vertical profiles of moisture and temperature were measured with a tethersonde $3.5 \mathrm{~km}$ from the slope. Early morning profiles showed roughly constant lapse rates up to about $1000 \mathrm{~m}$, except very near the surface. Rangeheight indicator (RHI) scans with a lidar provided information on the backscatter boundary layer (BBL) depth over plain and slope, which reached values of about $800 \mathrm{~m}$. Over the plain close to the slope, (at the origin in Fig. 1a) a Doppler sodar was employed to measure vertical profiles of wind velocity averaged over approximately 20 minutes. 'Upslope flow velocities' were determined as the maximum value of the horizontal along slope wind component, typically 3-6 $\mathrm{m} \mathrm{s}^{-1}$. The approximately linear background stratification and simple geometry at Minnekhada Park are well suited for idealizations in a water-tank model.

\section{b. Water Tank}

The water tank (Fig. 1b) has glass walls and a stainless steel bottom encased in a stainless steel frame. Because field observations showed fairly constant lapse rate all water-tank experiments were started with linear salt stratification. Convection is triggered in the tank by heating the bottom steel plate from below with strip heaters. Because heating could only be varied manually within $40-100 \%$ of the maximum power supply, most experiments were carried out with steady heat flux. This paper focuses on three experiments with two different parameter settings. Two water-tank experiments ('WS') with identical settings had steady surface heating, and parameters were chosen to achieve similarity with observations at Minnekhada Park at 1100 PST (Pacific Standard Time, which is within 15 minutes of local solar time and corresponds to 1900 UTC) on 25 July 2001. The BBL depth was determined in the first experiment from photographs and videos of fluorescent dye, originally released as a thin dense layer over the plain; the BBL reached depths of about $15 \mathrm{~cm}$. In the second experiment, two-dimensional velocity fields were measured from the motion of neutrally buoyant particles using the PIV ('particle image velocimetry') toolbox for Matlab. A third control experiment ('WC'), also with neutrally buoyant particles, employed manually controlled heat fluxes to approximate the roughly sinusoidal surface heat flux development found in the atmosphere. Maximum upslope flow velocities were computed in two steps. First, medians over 20-s intervals were calculated to remove turbulent variations. Medians successfully removed outliers and were faster to calculate than means with outlier filters. Second, the medians of the maxima in three adjacent vertical profiles of horizontal velocities centered over the slope $20 \mathrm{~cm}$ from the slope base were calculated to further smooth the data. The use of the median rather than the mean slightly reduced some extreme values. The results were very robust when averaging time was doubled and when five rather than three adjacent profiles were used. Experiments lasted roughly $1000 \mathrm{~s}$. Maximum horizontal velocities ranged from $0.5-2.0 \mathrm{~cm} \mathrm{~s}^{-1}$. More technical details can be found in [10]. The water-tank parameters are provided in the next section as part of the scaling.

In this paper, quantities specific to the atmosphere will be distinguished by a subscript ' $a$ ' from water-tank specific quantities, which will carry subscript 'w'. Further discriminations are applied, as necessary, for the two watertank experiments with steady surface heat flux ('ws') and the control experiment with sinusoidal heat flux ('wc'). If a quantity applies generally to atmosphere and water tank the subscript will be dropped.

\section{SCALING}

The range of BBL depths and upslope flow velocities during the course of a tank experiment or the morning hours at Minnekhada Park (roughly an order of magnitude) is much greater than the uncertainties of field and tank observations at a given point in time (about 20\%). To make use of the smaller uncertainties in the observations, scaling must consider the time dependence of heating and all idealizations. Fig. (2) demonstrates the approach that physical scale modelers follow implicitly. The real physical systems (atmosphere at Minnekhada Park and the water tank) are not compared directly but through their mathematical idealizations. A quantitative link between observations in atmosphere and water tank is established in four steps: (i) Based on the observations, mathematical idealizations of atmosphere and water tank are developed: 'atmospheric idealization' (AI) and 'water-tank idealization' (WTI) ('Data' arrows in Fig. 2). This will be discussed in 
(a)

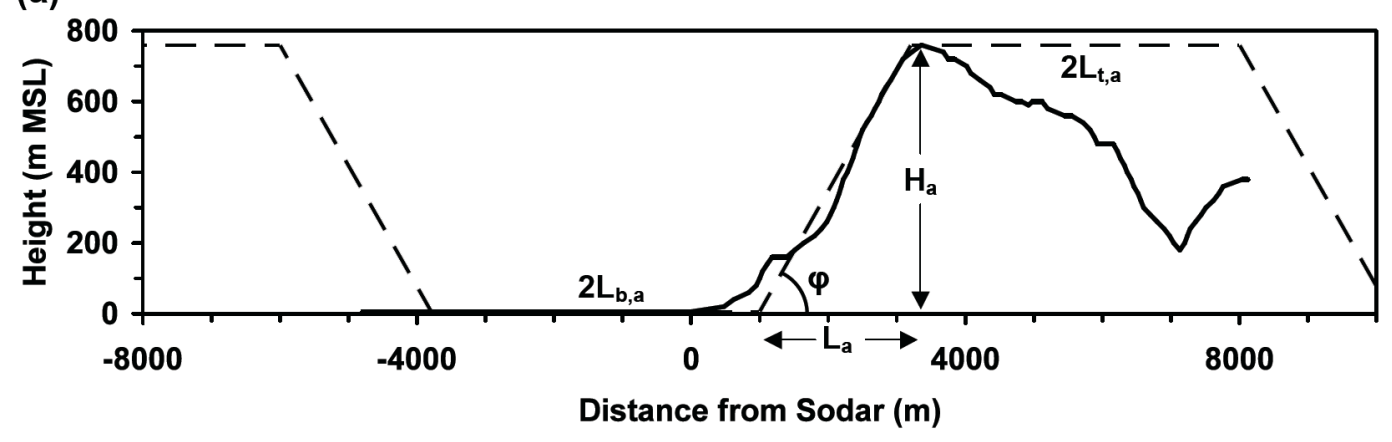

(b)

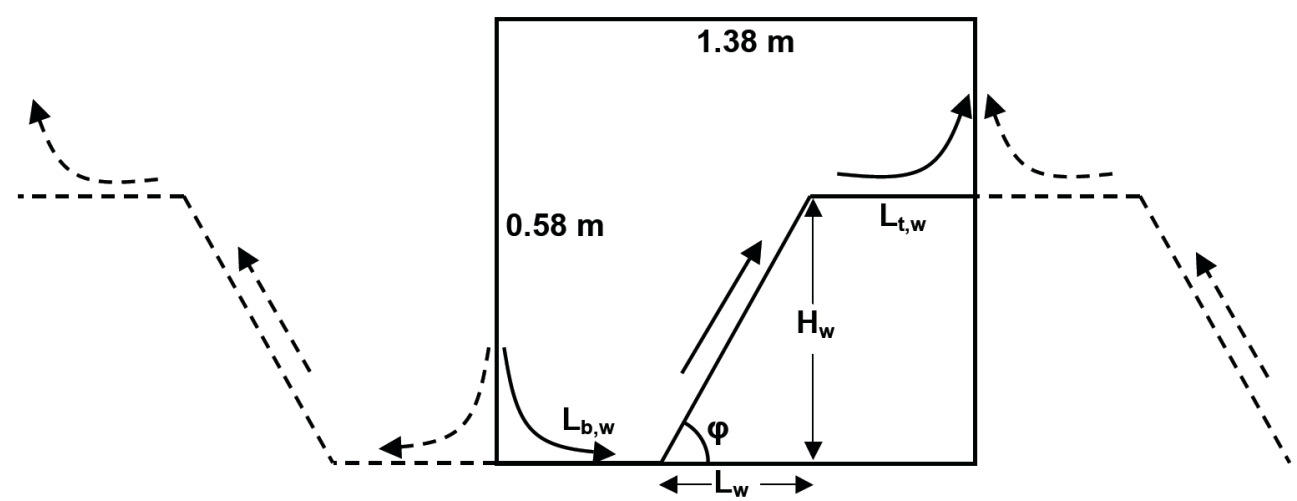

Fig. (1). Topography at the field site and atmospheric and water-tank idealizations. (a) The solid line shows the vertical cross-section of the slope at the field site. The dashed line represents the idealized periodic topography. (b) The solid box encloses the water-tank domain. The end walls impose a mirror symmetry shown by a schematic flow pattern within the tank (solid arrows) and the imaginary mirrored flow outside the tank (dashed arrows). Notice that horizontal and vertical axes do not have the same scale.

section a. (ii) In section b, ND governing parameters ('Pi groups') are determined for the two idealizations, AI and WTI, using the Buckingham Pi theorem (Buckingham 1914). (iii) The link between atmosphere and water tank is completed by imposing similarity constraints on the $\mathrm{Pi}$ groups in AI and WTI (section c). Differences in surface heat fluxes, sinusoidal versus steady, make the scaling implicitly time-dependent which is further discussed. (iv) To test the similarity between atmosphere and water tank, the Pi groups and further assumptions are used to predict field and tank observations of BBL depth and upslope flow velocity ('Prediction' arrows in Fig. 2), covered in section 4.

\section{a. Atmospheric and Water-Tank Idealizations}

The first step is to develop the mathematical idealizations, AI and WTI, from the data ('Data' arrows in Fig. 2). The topography of the AI is infinite in the crossslope direction and consists of an infinite periodic array of plains at mean sea level (Fig. 1a) with half length
$L_{b, a}=2400 \mathrm{~m}$

and plateaus at

$H_{a}=760 \mathrm{~m} \mathrm{MSL}$

with half length

$L_{t, a}=2400 \mathrm{~m}$

separated by slopes with a horizontal length of

$L_{a}=2207 \mathrm{~m}$.

In the corresponding WTI (Fig. 1b),

$L_{b, w}=L_{t, w}=0.470 \mathrm{~m}$,

$H_{w}=0.149 \mathrm{~m}$, and

$L_{w}=0.433 \mathrm{~m}$.

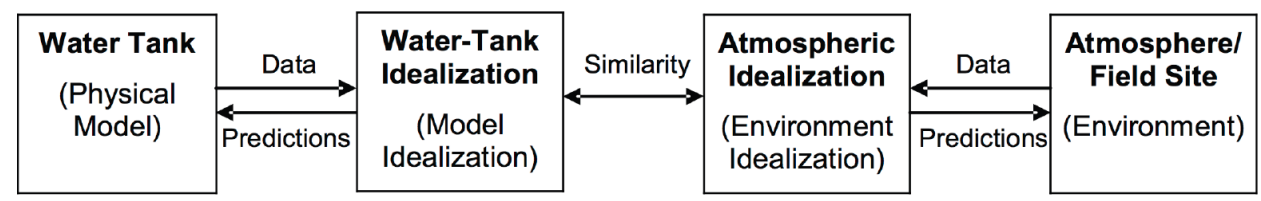

Fig. (2). Concept map of the scaling. Scaling has to be carried out between each pair of neighbouring boxes. The atmosphere and the water tank provide data to develop atmospheric and water-tank idealizations, respectively. In return, the two idealizations can be used to predict other quantities in the real atmosphere and the water tank. Central is the similarity over many orders of magnitude between atmospheric idealization and water-tank idealization. 
$W_{w}=0.431 \mathrm{~m}$ and

$D_{w}=0.580 \mathrm{~m}$.

In both AI and WTI, the aspect ratio $L_{w} / H_{w}=2.90$ corresponds to a slope angle $\varphi=19^{\circ}$. Plain and plateau lengths $L_{b, w}$ and $L_{t, w}$ are constraints originating from the finite dimensions of the tank. The tank's end walls impose a mirror or even-parity symmetry indicated by the schematic flow patterns inside and outside of the water-tank domain (Fig. 1b) if heat loss and friction between the end wall and the fluid are neglected [11]. The corresponding parameters of the AI, $L_{b, a}$ and $L_{t, a}$ in (1) and (3), are chosen such that AI and WTI are geometrically similar, that is

$\frac{L_{b, a}}{H_{a}}=\frac{L_{b, w}}{H_{w}}$ and $\frac{L_{t, a}}{H_{a}}=\frac{L_{t, w}}{H_{w}}$.

The other physical characteristic of the slope that should be considered is its smoothness, or alternatively, the size of the roughness elements, $Z_{r}$. At the field site, roughness elements ranged from metres for bushes, hedges, and buildings to tens of metres for tall trees. The well-sanded and painted tank bottom lacks any roughness elements of more than a few tenth of a millimetre.

As for sea breezes (e.g. [12]), the buoyancy parameters

$g \beta_{a} \approx 0.036 \mathrm{~m} \mathrm{~s}^{-2} \mathrm{~K}^{-1}$ and $g \beta_{w} \approx 2.59 \times 10^{-3} \mathrm{~m} \mathrm{~s}^{-2} \mathrm{~K}^{-1}$

should be important in upslope flows. Here, $g$ is the gravitational acceleration and $\beta$ the coefficient of thermal expansion. The choice of the product $g \beta$ rather than individual $g$ and $\beta$ as governing parameters is equivalent to making the Boussinesq approximation [3].

In the AI, the air is assumed dry and linearly stably stratified at the beginning of positive net sensible surface heat flux, hence for AI and WTI a constant initial background buoyancy frequencies (Brunt-Väisälä frequencies) is assumed:

$N_{a}=\left(g \beta_{a} \frac{d \theta_{a}}{d z_{a}}\right)^{1 / 2}$ and $N_{w}=\left(\frac{g}{\alpha_{0, w}} \frac{d \alpha_{w}}{d z_{w}}\right)^{1 / 2}$,

where $z$ is the height above ground and $\theta_{a}$ is the background potential temperature in $\mathrm{AI} ; \alpha_{w}$ is the background specific volume in WTI defined as the inverse of density $\rho_{w}$ :

$\alpha_{w} \equiv \frac{1}{\rho_{w}}=\frac{\delta V_{w}}{\delta m_{w}}$,

with $\delta V_{w}$ the volume and $\delta m_{w}$ the mass of a test parcel. Finally, $\alpha_{0, w} \approx 1.0018 \times 10^{-3} \mathrm{~m}^{3} \mathrm{~kg}^{-1}$.

When requiring similarity between AI and WTI, advection and subsidence external to the slope system and the Coriolis force must be neglected due to technical limitations of the water tank. In the AI it is assumed that surface roughness and heating are homogeneous and that sensible surface heat flux depends sinusoidally on time $t_{a}$ :

$Q_{H, a}\left(t_{a}\right)=Q_{\max , a} \cdot \sin \left(\frac{\pi}{2} \frac{t_{a}}{t_{d, a}}\right)$,

where maximum sensible heat flux $Q_{\max , a} \approx 0.289 \mathrm{~K} \mathrm{~m} \mathrm{~s}^{-1}$ and diurnal heating time scale $t_{d, a} \approx 7.75 \mathrm{~h}=27900 \mathrm{~s}$ were extracted from an ensemble average of heat flux measurements taken in the Lower Fraser Valley on individual days in the interval from 17 July - 26 August over the years 1983-1986 (see figure 8 in [12]). The beginning of positive surface heat flux was approximately two and a half hours after sunrise at 0700 PST, and maximum heat flux was reached at about 1445 PST.

Instantaneous heat flux $Q_{H, a}$ drives convection. In addition, time-integrated heat flux, which is the total supplied energy per surface area (energy density), is an important quantity that for example determines the BBL depth. For the AI,

$E_{a}=\int_{0}^{t_{a}} Q_{H, a}(t) d t=Q_{\max , a} t_{d, a} \cdot \frac{2}{\pi}\left[1-\cos \left(\frac{\pi}{2} \frac{t_{a}}{t_{d, a}}\right)\right]$.

In the water tank, the control experiment WC was carried out with roughly sinusoidal heat flux so that (14) and (15) apply with subscripts 'a' replaced by 'wc' and with parameters $Q_{\mathrm{max}, w c} \approx 3.7 \times 10^{-3} \mathrm{~K} \mathrm{~m} \mathrm{~s}^{-1}$ and $t_{d, w c} \approx 974 \mathrm{~s}$ :

$Q_{H, w c}\left(t_{w c}\right)=Q_{\text {max }, w c} \cdot \sin \left(\frac{\pi}{2} \frac{t_{w c}}{t_{d, w c}}\right)$ and

$E_{w c}=Q_{\mathrm{max}, w c} t_{d, w c} \cdot \frac{2}{\pi}\left[1-\cos \left(\frac{\pi}{2} \frac{t_{w c}}{t_{d, w c}}\right)\right]$.

In the two experiments WS with steady surface heat flux:

$Q_{H, w s} \approx 1.85 \times 10^{-3} \mathrm{~K} \mathrm{~m} \mathrm{~s}^{-1}$ and

$E_{w s}=\int_{0}^{t_{w}} Q_{H, w s}(t) d t=Q_{H, w s} t_{w s}$,

where $t_{w s}$ is the water-tank reference time since the onset of heating in the water tank. Specifying the molecular parameters completes the description of AI and WTI: kinematic viscosities

$v_{a} \approx 1.52 \times 10^{-5} \mathrm{~m}^{2} \mathrm{~s}^{-1}$ and $v_{w} \approx 8.9 \times 10^{-7} \mathrm{~m}^{2} \mathrm{~s}^{-1}$,

and thermal diffusivities

$\kappa_{a} \approx 2.11 \times 10^{-5} \mathrm{~m}^{2} \mathrm{~s}^{-1}$ and $\kappa_{w} \approx 1.45 \times 10^{-7} \mathrm{~m}^{2} \mathrm{~s}^{-1}$.

The independent parameters for AI and WTI are summarized in Table $\mathbf{1}$.

\section{b. Derivation of Pi Groups}

The second step is to determine the Pi groups for AI and WTI. Table 1 lists $n=11$ independent parameters for the AI: 
Table 1. Independent Parameters in AI and WTI. The Distinguishing Subscript ' $a$ ' for AI and ' $w$ ' for WTI was Dropped for those Parameters that Apply to Both Idealizations

\begin{tabular}{|c|c|c|c|c|}
\hline Name & Symbol & Units & AI & WTI \\
\hline Ridge height & $H$ & $\mathrm{~m}$ & 760 & 0.149 \\
\hline Instantaneous heat flux & $Q_{H}$ & $\mathrm{~K} \mathrm{~m} \mathrm{~s}^{-1}$ & $0-0.289^{\mathrm{a}}$ & $0.0015-0.0037^{b}$ \\
\hline Buoyancy frequency & $N$ & $\mathrm{~s}^{-1}$ & $0.0149-0.0162^{\mathrm{c}}$ & $0.1-1.5^{b}$ \\
\hline Horizontal slope length & $L$ & $\mathrm{~m}$ & 2207 & 0.433 \\
\hline Energy density & E & $\mathrm{K} \mathrm{m}$ & $0-5130^{\text {a }}$ & $0-6^{\mathrm{b}}$ \\
\hline Buoyancy parameter & $g \beta$ & $\mathrm{m} \mathrm{s}^{-2} \mathrm{~K}^{-1}$ & 0.036 & 0.00259 \\
\hline Kinematic viscosity & $v$ & $\mathrm{~m}^{2} \mathrm{~s}^{-1}$ & $1.52 \times 10^{-5}$ & $8.9 \times 10^{-7}$ \\
\hline Thermal diffusivity & $\kappa$ & $\mathrm{m}^{2} \mathrm{~s}^{-1}$ & $2.11 \times 10^{-5}$ & $1.45 \times 10^{-7}$ \\
\hline Tank width & $W_{w}$ & $\mathrm{~m}$ & - & 0.431 \\
\hline Water depth & $D_{w}$ & $\mathrm{~m}$ & - & 0.580 \\
\hline
\end{tabular}

${ }^{\mathrm{a}}$ Dependent on time of day.

${ }^{\mathrm{b} C}$ Controllable.

${ }^{c}$ Dependent on day.

$H_{a}, Q_{H, a}, N_{a}, L_{a}, Z_{r}, E_{a}, g \beta_{a}, v_{a}, \kappa_{a}, L_{b, a}$, and $L_{t, a}$. These use $k=3$ fundamental units $(\mathrm{K}, \mathrm{m}, \mathrm{s})$. According to the Buckingham Pi Theorem [5], $k=3$ independent key parameters are needed to form $n-k=8$ ND governing parameters (Pi groups). A suitable choice are the first three dimensionally independent parameters with their units shown in brackets: $H_{a}[\mathrm{~m}], Q_{H, a}\left[\mathrm{~K} \mathrm{~m} \mathrm{~s}^{-1}\right]$, and $N_{a}\left[\mathrm{~s}^{-1}\right]$. Pi groups can be formed by non-dimensionalizing the remaining eight variables $L_{a}, E_{a}, g \beta_{a}, v_{a}, \kappa_{a}, L_{b, a}, L_{t, a}$, and $Z_{r}$ with appropriate combinations of the key variables. This leads to the first eight of the Pi groups in Table 2. In the WTI, there are two additional independent parameters, tank width $W_{w}$ and water depth $D_{w}$. Because the number of fundamental units, $k=3$, is the same as in the AI (Table $\mathbf{1}$ ), two additional Pi groups, $\Pi_{8, w}$ and $\Pi_{9, w}$, are required to completely describe the WTI. Note that $\Pi_{2, w c}=N_{w c} t_{w c}$, therefore $\Pi_{2}$ can be interpreted as ND time.

The ten Pi groups fall into five categories (Table 2). A reasonable first guess is that the 'core Pi groups', $\Pi_{1}$ to $\Pi_{3}$, guarantee similarity between AI and WTI for bulk flow features. For the AI, bulk flow features occur roughly at time scales of $O(\geq 10 \mathrm{~min})$, horizontal length scales of $O(\geq 100 \mathrm{~m})$, and vertical length scales of $O(\geq 20 \mathrm{~m})$. Bulk flow features should not be affected by the 'molecular Pi groups', $\Pi_{4}$ and $\Pi_{5}$, as long as the flow is fully turbulent. This latter assumption is often expressed as requiring the Reynolds number to exceed a critical value. Chen et al. [7] use $\operatorname{Re}=U L / v$. Using the observed velocities in our experiments, the corresponding Reynolds numbers are roughly $2.6 \times 10^{3}$, about a factor of three greater than [7]. Following their arguments, this value should scale well with the atmospheric values based on typical eddy viscosities. On the other hand [13], suggested basing the Reynolds number on the geometry of open channel flows and found values of about 500 , well below the range of intermittently turbulent flow from 1350-1800 [14]. The scaling used in this paper circumvents difficulties in defining the velocity and length scales of the flow and uses $\Pi_{4}$ and $\Pi_{5}$ to define the effect of molecular quantities.

The third category, 'longitudinal boundary conditions', $\Pi_{6}$ and $\Pi_{7}$, is a consequence of the finite length of the water tank, which imposes the symmetry restriction on the AI (Fig. 1). Tank observations using variations in the length of the plain and plateau [10] show that the slope flow system is confined to an area close to the slope so that $\Pi_{6}$ and $\Pi_{7}$ are large enough for the upslope flow system to be independent of the particular values. Finally, the 'water tankspecific Pi groups', $\Pi_{8, w}$ and $\Pi_{9, w}$, are assumed to be asymptotically large enough to neglect the influence of the side walls and the finite depth of the water. The assumptions made in this paragraph will be discussed in section 6 .

Lastly $\Pi_{10}$, the roughness height, is of the same order but slightly larger in the tank. Schumann [8] and Hunt et al. [9] found very weak dependencies on the momentum roughness length, which is a function of the height and distribution of roughness elements. This only weak dependence would give a discrepancy between the tank and the atmosphere of less than a few tenth of a percent. We will revisit this in the discussion (section 5). 
Table 2. Summary of Pi Groups in AI and WTI. The Distinguishing Subscript ' $a$ ' for AI and 'w' for WTI was Dropped for those Pi Groups that Apply to Both Idealizations

\begin{tabular}{|c|c|}
\hline Name & Symbol \\
\hline \multicolumn{2}{|c|}{ Core Pi Groups } \\
\hline Aspect ratio & $\Pi_{1}=\frac{L}{H}$ \\
\hline ND energy density or time & $\Pi_{2}=\frac{E N}{Q_{H}}$ \\
\hline ND buoyancy parameter / heat flux & $\Pi_{3}=\frac{g \beta Q_{H}}{H^{2} N^{3}}$ \\
\hline \multicolumn{2}{|c|}{ Molecular Pi Groups } \\
\hline ND viscosity & $\Pi_{4}=\frac{v}{H^{2} N}$ \\
\hline ND thermal diffusivity & $\Pi_{5}=\frac{\kappa}{H^{2} N}$ \\
\hline \multicolumn{2}{|c|}{ Longitudinal Boundary Conditions } \\
\hline ND half length of plain & $\Pi_{6}=\frac{L_{b}}{H}$ \\
\hline ND half length of plateau & $\Pi_{7}=\frac{L_{t}}{H}$ \\
\hline \multicolumn{2}{|l|}{ Water-tank specific Pi groups } \\
\hline ND tank width & $\Pi_{8, w}=\frac{W_{w}}{H_{w}}$ \\
\hline ND tank depth & $\Pi_{9, w}=\frac{D_{w}}{H_{w}}$ \\
\hline \multicolumn{2}{|c|}{ Roughness Length Pi Groups } \\
\hline ND roughness height & $\Pi_{10}=\frac{Z_{r}}{H}$ \\
\hline
\end{tabular}

\section{c. Similarity Between Atmospheric and Water-Tank Idealizations}

In the third step, the link in the concept map (Fig. 2) between atmosphere and water tank is completed by imposing similarity constraints on the Pi groups of AI and WTI. Similarity of the bulk features in AI and WTI, requires:

$\Pi_{1, a}=\Pi_{1, w}$,

$\Pi_{2, a}=\Pi_{2, w}$, and

$\Pi_{3, a}=\Pi_{3, w}$.

The aspect ratio $\Pi_{1}$ is equal for AI and WTI by design of the water tank. It is not a priori obvious that it is technically possible to design water-tank experiments that meet the similarity requirements. To verify this ND quantities are converted back to dimensional quantities. Of the original set of twelve independent quantities of the WTI, $H_{w}, Q_{H, w}$, $N_{w}, L_{w}, E_{w}, g \beta_{w}, v_{w}, \kappa_{w}, L_{b, w}, L_{t, w}, W_{w}$, and $D_{w}$ (Table 1), the last six quantities, $v_{w}, \kappa_{w}, L_{b, w}, L_{t, w}, W_{w}$, and $D_{w}$, are not affected by requiring similarity of bulk flow features. The first three independent parameters, ridge height $H_{w}$, horizontal slope length $L_{w}$, and buoyancy parameter $g \beta_{w}$ are fixed quantities that cannot be manipulated. The remaining three parameters, $Q_{H, w}, N_{w}$, and $E_{w}$, are constrained by the two equations, (23) and (24) so that one quantity remains independent. We chose $Q_{H, w}$, because of its narrow range of controllability. Furthermore, time can be measured more directly than energy density, so (17) and (19) were used to replace $E_{w}$ by $t_{w}$. The tank experiments with sinusoidal heating, WC, and steady heating, WS, are treated separately.

For WC to be similar to atmospheric observations, (14)(17) are substituted into the definitions of $\Pi_{2}$ and $\Pi_{3}$ in Table 2:

$$
\begin{aligned}
& \Pi_{2}=\frac{E N}{Q_{H}}=t_{d} N \frac{2}{\pi} \frac{1-\cos \left(\frac{\pi}{2} \frac{t}{t_{d}}\right)}{\sin \left(\frac{\pi}{2} \frac{t}{t_{d}}\right)} \\
& \Pi_{3}=\frac{g \beta Q_{H}}{H^{2} N^{3}}=\frac{g \beta Q_{\max }}{H^{2} N^{3}} \sin \left(\frac{\pi}{2} \frac{t}{t_{d}}\right)
\end{aligned}
$$

For (23) and (24) to apply, the arguments and amplitudes of the trigonometric functions must be equal, which results in

$$
\begin{aligned}
& N_{w c}=N_{a} \cdot\left(\frac{g \beta_{w} Q_{\text {max }, w c} H_{a}^{2}}{g \beta_{a} Q_{\text {max }, a} H_{w}^{2}}\right)^{1 / 3} \approx 0.4289 \mathrm{~s}^{-1} \text { and } \\
& t_{d, w c}=\frac{N_{a}}{N_{w c}} t_{d, a} \approx 974 \mathrm{~s}
\end{aligned}
$$

where we used the parameter settings from section a and $Q_{\max , w c} \approx 3.7 \times 10^{-3} \mathrm{~K} \mathrm{~m} \mathrm{~s}^{-1}$. Similarity between $\mathrm{WC}$ and the atmosphere is achieved at times

$$
t_{w c, s i m}=\frac{t_{d, w c}}{t_{d, a}} t_{a, s i m},
$$

where $t_{a, s i m}$ denotes the atmospheric reference time of similarity.

In WS surface heating is steady, so instantaneous heat flux and energy density grow differently from the atmosphere. Similarity is possible only at one instant in time for any given experimental configuration. Substituting (14), (15), (18), and (19) into the definitions of $\Pi_{2}$ and $\Pi_{3}$ in Table 2 gives 
$N_{w s}=N_{a}\left(\frac{g \beta_{w} Q_{H, w s} H_{a}^{2}}{g \beta_{a} Q_{\max , a} H_{w}^{2}}\right)^{1 / 3}\left[\sin \left(\frac{\pi}{2} \frac{t_{a, s i m}}{t_{d, a}}\right)\right]^{-1 / 3}$ and

$t_{w s, s i m}=\frac{N_{a}}{N_{w s}\left(t_{a, s i m}\right)} \cdot t_{d, a} \cdot \frac{2}{\pi}\left[1-\cos \left(\frac{\pi}{2} \frac{t_{a, s i m}}{t_{d, a}}\right)\right] \cdot\left[\sin \left(\frac{\pi}{2} \frac{t_{a, s i m}}{t_{d, a}}\right)\right]^{-1}$,

where the notation $N_{w s}\left(t_{a, s i m}\right)$ emphasizes that $N_{w s}$ is a function of $t_{a, s i m}$ through (30). All quantities on the righthand side in (30) are defined, and $N_{w s}$ is fully determined. This value needs to be chosen before the experiment (Fig. 3a). To achieve similarity for another instant in time, $t_{a, s i m}$, on the same day (in the atmosphere), another experiment with another $N_{w s}$ needs to be performed.
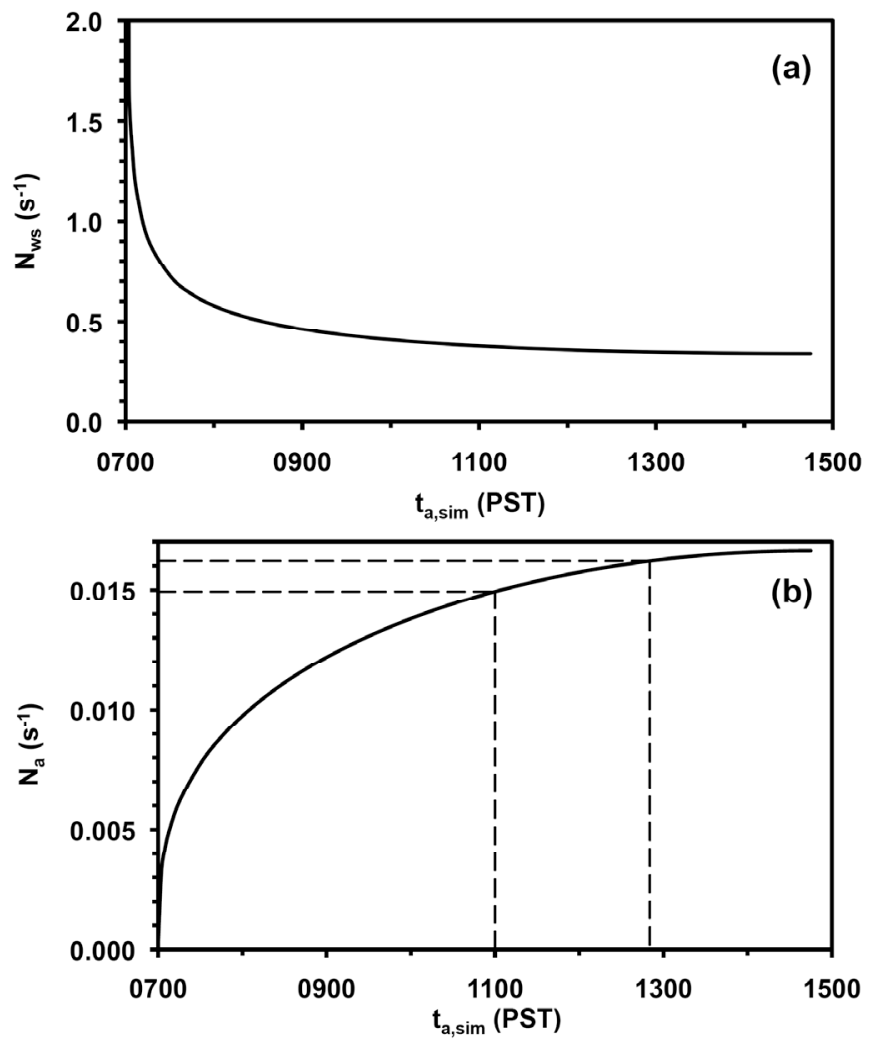

Fig. (3). (a) Water-tank buoyancy frequency $N_{w s}$ to be prepared at the beginning of WS to achieve similarity with atmospheric observations on 25 July $2001\left(N_{a}=0.0149 \mathrm{~s}^{-1}\right)$ at an instant in time, $t_{a, s i m}$. (b) Relation between atmospheric background buoyancy frequency $N_{a}$ and time of similarity $t_{a, s i m}$ for WS (solid line). The horizontal dashed lines mark background conditions on 25 and 26 July 2001. The vertical dashed lines show the corresponding time of day at which similarity between atmosphere and WS can be achieved.

An important corollary of the last two equations is: The same experiment with initial background buoyancy frequency $N_{w s}$ can be used to check similarity with atmospheric observations on different field days with different background buoyancy frequencies $N_{a}$. The times of similarity in atmosphere, $t_{a, s i m}$, and water tank, $t_{w s, s i m}$, follow from (30) and (31) (Fig. 3b).

\section{RESULTS}

With the first three steps of the scaling completed, similarity between atmosphere and water tank can be tested. It is assumed that the mathematical idealizations AI and WTI are good proxies for field observations in the morning of July 25, 2001, and for tank observations at corresponding times of similarity. Below, the $\mathrm{Pi}$ groups and further assumptions are used to predict field and tank observations of BBL depth and upslope flow velocity ('Prediction' arrows in the concept map, Fig. 2).

\section{a. Boundary-Layer Depth}

With the choice of key parameters $H, Q_{H}$, and $N$ (section b), ND BBL depth is

$h^{*}=\frac{h}{H}$

in AI and WTI. If most of the energy supplied by surface heating is converted into the growth of a vertically homogeneous BBL (after averaging over short-term variations caused by overshooting thermals and entrainment), the BBL depth depends only on the total supplied energy, but not on the instantaneous heat flux $Q_{H}$. Therefore, the ND BBL depth should only depend on

$\Pi_{2} \cdot \Pi_{3}=\frac{g \beta E}{H^{2} N^{3}}$,

which is the only combination of $\Pi_{2}$ and $\Pi_{3}$ that does not contain $Q_{H}$. To achieve similarity of BBL depth between atmosphere and water tank, that is $h_{a}{ }^{*}=h_{w}{ }^{*}$, it therefore suffices to require

$\Pi_{2, a} \cdot \Pi_{3, a}=\Pi_{2, w} \cdot \Pi_{3, w}$.

Similarity between atmosphere and water tank can be tested with one tank experiment at many points in time, because substituting (15) and (19) into (33) and (34) gives

$t_{w}=t_{d, a} \cdot \frac{2}{\pi}\left(\frac{H_{w} N_{w}}{H_{a} N_{a}}\right)^{2} \frac{\beta_{a} Q_{\max , a}}{\beta_{w} Q_{H, w}}\left[1-\cos \left(\frac{\pi}{2} \frac{t_{a}}{t_{d, a}}\right)\right]$.

For every atmospheric observation of BBL depth $h_{a}$ the corresponding ND BBL depth $h_{a}{ }^{*}$ can be calculated from (32), and the time $t_{a}$ of the observation can be converted to a corresponding water-tank reference time $t_{w}$ using (35). These values can be directly compared with ND BBL in the water tank, $h_{w}{ }^{*}$, using measurements of $h_{w}$ at water-tank reference time $t_{w}$ (Fig. 4).

Between $t_{w}=45 \mathrm{~s}$ and $t_{w}=240 \mathrm{~s}$, corresponding to 1036-1141 PST, ND BBL depths in atmosphere and water tank agree well. After $t_{w}=240 \mathrm{~s}$, lidar observations are roughly $20 \%$ higher than water-tank observations, caused by 
the onset of sea-breeze and up-valley flow near noon in the atmosphere, an effect that was not included in the AI [10].

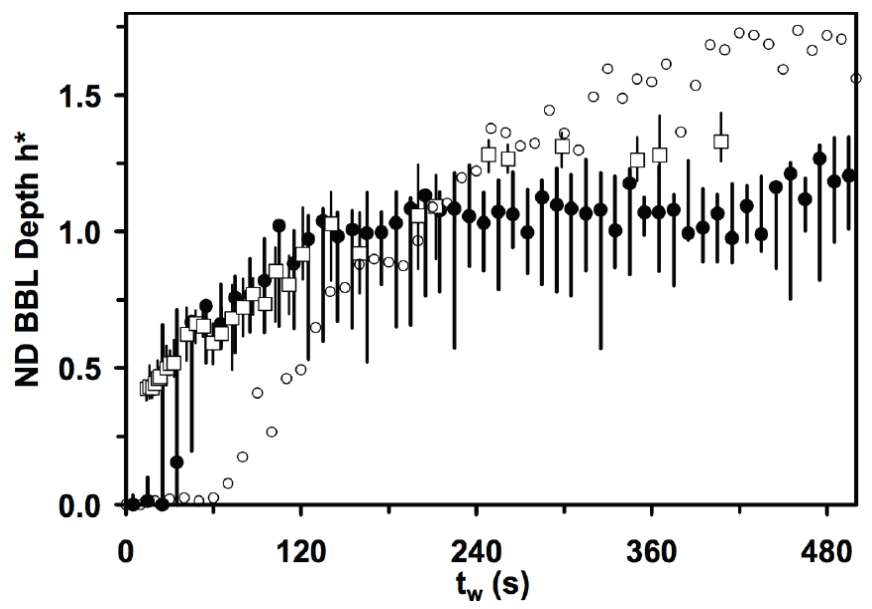

Fig. (4). Observations of ND BBL depth $h^{*}$ in atmosphere (open squares) and water tank (solid circles) over the plain near the slope base. Atmospheric observations are determined from lidar observations of the aerosol layer; tank observations are based on dispersed dye, which was originally released as a thin layer over the plain. Atmospheric reference time $t_{a}$ was converted into water-tank reference times $t_{w}$ using (35). Error bars indicate minimum and maximum BBL depths within a horizontal range of approximately $750 \mathrm{~m}$ (atmosphere) and $0.15 \mathrm{~m}$ (water tank). Parameters were $Q_{H, w}=1.85 \times 10^{-3} \mathrm{Kms}^{-1}, \quad N_{w}=0.379 \mathrm{~s}^{-1}$, and all others as in section 3.a. Open circles are tank observations of ND BBL depth over the heated plateau, only (separated from the rest of the tank), for a slightly weaker buoyancy frequency $N_{w}=0.324 \mathrm{~s}^{-1}$.

For comparison, Fig. (1) also shows observations of ND BBL depth for a flat horizontal surface (over the plateau of the water tank with the remainder of the tank separated by a wall) for slightly weaker background stratification. Independent of the particular position shown in Fig. (4), the BBL over a flat surface initially grows more slowly than under the influence of a slope, but it continues growing monotonously and after $220 \mathrm{~s}$ is deeper than under the influence of a slope. By contrast, under the influence of a slope, the BBL initially grows very fast, but shortly after 120 $\mathrm{s}$, when the $\mathrm{BBL}$ reaches approximately ridge height, i.e. $h^{*} \approx 1$, it remains constant until about $450 \mathrm{~s}$, when it starts deepening again. Time development of BBL depth in the tank under the presence of the slope is more similar to the field observations than to the tank observations without a slope. This indicates that the presence of the slope substantially alters the growth of the BBL and that the watertank experiment captures this difference.

\section{b. Time}

BBL depth is time dependent via its dependence on integrated heat flux but is similar in atmosphere and experiment WS within about $20 \%$ uncertainty. This suggests that the different time dependences of surface heat fluxes in atmosphere and water tank do not lead to similarity violations. However, similarity of BBL depth in experiment WS and the atmosphere requires only equality of the product $\Pi_{2} \cdot \Pi_{3}$. In contrast, maximum upslope flow velocity requires equality of the individual $\mathrm{Pi}$ groups, which is possible only at one point in time according to (31). Maximum upslope flow velocities in WS and WC are compared, first. This permits testing of the time dependence without other potential sources of similarity violations that could potentially arise when tank experiment WS is compared with the atmospheric observations. (30) and (31) apply with subscript 'a' replaced by 'wc'.

Fig. (5) shows the surface heat flux in WC as compared to the ideal sinusoidal time development. Although this approximation is crude, it is very different from a steady heat flux. The tank parameters $N_{w c}=0.4289 \mathrm{~s}^{-1}, t_{d, w c}=974 \mathrm{~s}$, and $Q_{\max , w c}=0.0037 \mathrm{~K} \mathrm{~m} \mathrm{~s}^{-1}$ in WC were chosen to achieve similarity with WS at $t_{w c, s i m}=502 \mathrm{~s}$ as indicated by the dashed line in Fig. (5). Before and after this time, velocity similarity cannot be expected.

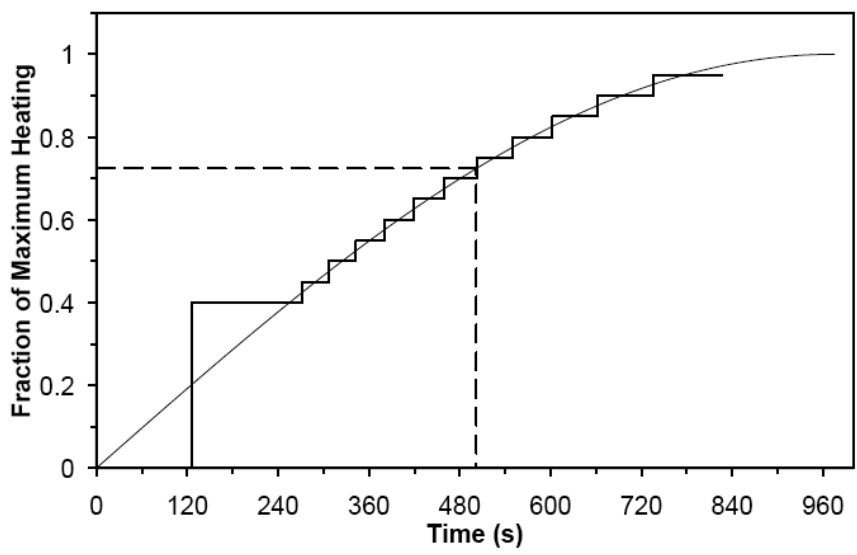

Fig. (5). Heat flux profile of control experiment (thick line with discrete steps). The experiment terminated at 826 seconds when a thermal breaker, prohibiting extended power draw beyond $85 \%$ of the maximum allowable power for the heaters, tripped. The initial rapid increase at 130 seconds was necessary because the controls did not allow less than $40 \%$ power supply. The thin line shows the sinusoidal heating approximated by the stepped curve in such a way that the area underneath both curves (total supplied energy) is approximately equal at any given time after 270 seconds. The dashed curve shows the time of similarity between water tank and atmosphere for the atmospheric test case discussed in the main text.

Velocities are non-dimensionalized by dividing velocities by the two key parameters $H$ and $N$ :

$U^{*}=\frac{U}{H N}$

Fig. (6) shows that ND velocities in WC and WS begin to diverge before the time of expected similarity. This divergence may be due to large amplitude oscillations seen in both water tank experiments, which happen to be of opposite phase at $t_{w c, s i m}$. These oscillations are of interest per se but are outside the scope of this work. Despite the oscillations, the two tank experiments agree reasonably well at $t_{w c, s i m}$. At other times, when similarity cannot be expected, ND velocities in WC and WS differ, but never by more than a factor 2. This strongly contrasts ND velocities in the atmosphere, which will be discussed next. 


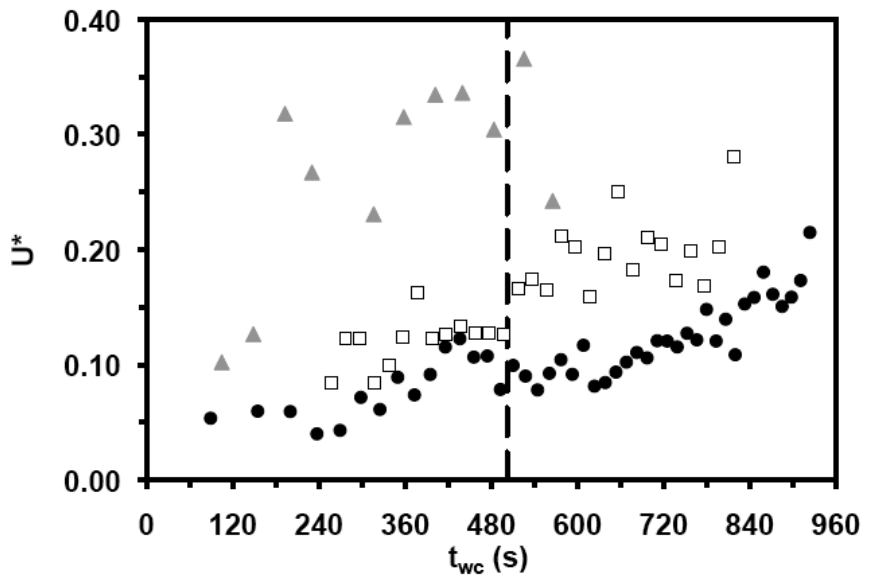

Fig. (6). Non-dimensional maximum upslope flow velocities in atmosphere (grey triangles), control experiment WC (open squares), and tank experiment with steady heat flux WS (black circles). Heating in the control experiment followed the stepped curve in Fig . (5). Observed values are medians over 20-second intervals centered at $t_{w c}$ of each data point and averages over the maxima in three adjacent vertical profiles of horizontal velocities over the slope midpoint. Times in the atmosphere, $t_{a}$, and in WS, $t_{w s}$, were transferred into the laboratory time $t_{w c}$ of the control experiment.

\section{c. Upslope Flow Velocity}

Fig. (6) shows that ND velocities are roughly two to four times greater in the atmosphere than in WC at almost all times. A similarly large discrepancy can be seen for ND velocities at the time of similarity with WS. Even much earlier, when instantaneous and integrated heat fluxes are relatively much greater in WS than in the atmosphere, ND velocities are about twice as large in the atmosphere as in WS. Reasonably close values in WS and WC (despite different heating profiles) and much larger values for the atmosphere (despite heating profiles similar to WC) suggest that velocity similarity between atmosphere and water tank is violated for reasons other than different time dependences of surface heat fluxes.

This completes the similarity test between atmosphere and water tank and the four steps of the scaling. The implications of these results will be discussed in the following section.

\section{DISCUSSION}

The results of the previous section provide evidence for the following conclusions. Firstly, BBL depths in atmosphere and water tank are equal within an uncertainty of about $20 \%$. Secondly, the exact history of surface heat flux is not important to find agreement between two tank experiments, only equality of the two Pi groups $\Pi_{2}$ and $\Pi_{3}$ is needed. Thirdly, ND upslope flow velocities in water tank and atmosphere are significantly different.

The first two conclusions demonstrate that quantitative scaling between water tank and atmosphere is possible within the $20 \%$ uncertainties of the field observations. This led to the third conclusion, similarity violation of upslope flow velocity. This latter result implies that more Pi groups need to be included in the scaling of upslope flow velocities. The finite dimensions of the tank, which are included in $\Pi_{6, w}$ to $\Pi_{9, w}$, do not seem to influence the flow patterns substantially until late in the water-tank experiments [10]. The molecular Pi groups $\Pi_{4}$ and $\Pi_{5}$ are unlikely to be directly responsible for the similarity violation. For example, an estimation of the various terms in the governing equations (for example [15]) shows that the molecular terms are much smaller than other terms. However, on the scale of the roughness height, molecular effects must be dominant in the tank. This could be expressed as $\Pi_{4} /\left(\Pi_{10}^{2}\right)$. Alternatively, we can estimate the roughness Reynolds number $\operatorname{Re}=u_{\tau} k / v \sim 0.1$, where friction velocity $u_{\tau} \sim 10^{-4} \mathrm{~m} \mathrm{~s}^{-1}$, height of roughness elements $k \sim 10^{-4} \mathrm{~m}$, and molecular viscosity $v \sim 10^{-6} \mathrm{~m}^{2} \mathrm{~s}^{-1}$. A roughness Reynolds number less than unity is a strong indication of fluiddynamical smoothness. An investigation of the role of surface roughness in the similarity violation requires a detailed discussion of alternative hypotheses for upslope flow velocity and an empirical analysis of additional water-tank data. Estimation of this role allows a selection of a scaling for upslope flow in the atmosphere; this is presented in a follow-on paper.

Previous water-tank studies have not mentioned the similarity violation of upslope flow velocity $[7,11,16]$. An order-of-magnitude estimation of the upslope flow velocity from our tank experiments taken at a later stage, for example at $t_{w c}=900 \mathrm{~s}$ (Fig. 6), produces "typical" atmospheric values of about $3 \mathrm{~m} \mathrm{~s}^{-1}$. However, it was demonstrated in section 4 that atmosphere and water tank do not meet the similarity requirements for these particular parameter values. We ran experiments as similar to these three previous studies as could be inferred from the available information. Following the same careful scaling as in this paper, these experiments also show velocity similarity violations.

Despite the similarity violation of upslope flow velocities, it was shown in [10, figure 12] that tank dye images and atmospheric lidar scans are qualitatively similar at the time of expected similarity. The aerosol/dye distributions in atmosphere and water tank seem mostly related to the BBL depth, which is similar in the two systems, and unaffected by the similarity violation of upslope flow velocities. Minor differences in the distributions can partially be attributed to differences in ND upslope flow velocities, but other factors play an equally important role.

\section{SUMMARY AND CONCLUSIONS}

In this paper, scaling of upslope flows in atmosphere and water tank is performed in four steps based on potentially relevant physical quantities and by explicitly distinguishing atmosphere and water tank from their mathematical idealizations. In the first step, the mathematical idealizations are developed and their governing parameters determined. In the second step, the Buckingham Pi Theorem is applied to determine the ND governing parameters (Pi groups). Next, similarity constraints are imposed on the $\mathrm{Pi}$ groups of the 
two mathematical idealizations. Finally, observable quantities in environment and physical model are derived based on the idealizations and further assumptions, and their similarity is tested.

Three core $\mathrm{Pi}$ groups are identified: the aspect ratio $\Pi_{1}=L / H$ (horizontal slope length divided by ridge height), the ND energy density or ND time $\Pi_{2}=E N / Q_{H}$, and the ND buoyancy parameter or ND instantaneous surface heat flux $\Pi_{3}=g \beta Q_{H} / H^{2} N^{3}$. Based on these $\mathrm{Pi}$ groups $\mathrm{BBL}$ depths in atmosphere and water tank were similar within $20 \%$. Time dependence was included by using both instantaneous surface heat flux, $Q_{H}$, and energy density (integrated surface heat flux), $E$, in the scaling. By comparing upslope flow velocities in two tank experiments, one with steady heat flux and one with approximately sinusoidally time dependent heat flux, it was shown that equality of the three core Pi groups suffices to achieve similarity of upslope flow velocities in the water tanks at one point in time, regardless of the specific history of surface heat flux. A comparison of both tank experiments with atmospheric observations showed that similarity of maximum upslope flow velocity in atmosphere and water tank was violated by a factor of more than two. However, the similarity violation does not cause substantially different dye/aerosol distributions. We speculate that differences in Reynolds number and roughness height between tank and atmosphere may be the cause for the velocity similarity violation. A second part to this paper will discuss the similarity violation in detail.

\section{ACKNOWLEDGEMENTS}

We thank Greg Lawrence for providing laboratory space for the water tank and sharing computer resources and instrumentation. The tank was built in the workshop in the Department of Civil Engineering at UBC: Bill Leung built the mechanical parts and Scott Jackson the electrical and electronic components. Workshop supervisor Harald Schrempp generously allocated workshop time, and his experience was of tremendous help particularly during the design stage. We used the Matlab PIV toolbox developed by J. Kristian Sveen to determine velocities in the water tank. Ian Chan was of tremendous help in re-designing and rebuilding the tank, re-writing parts of MatPIV, and running experiments. Funding support was provided by grants from NSERC and CFCAS to D. Steyn and S. Allen.

\section{REFERENCES}

[1] Whiteman CD. Mountain meteorology: fundamentals and applications; Oxford Univ Press: New York, USA 2000.

[2] Reuten C, Steyn DG, Strawbridge KB, Bovis P. Observations of the relation between upslope flows and the convective boundary layer in steep terrain. Bound-Layer Meteor 2005; 116: 37-61.

[3] Barenblatt GI. Scaling; Cambridge Univ Press: Cambridge, UK 2003.

[4] Tolman RC. The principle of similitude. Phys Rev Lett 1914; Second Series, III: 244-55.

[5] Buckingham E. On physically similar systems; illustrations of the use of dimensional equations. Phys Rev Lett 1914; Second Series, IV: $345-76$.

[6] Tritton DJ. Physical fluid dynamics. Oxford Univ Press: Oxford, UK 1988.

[7] Chen RR, Berman NS, Boyer DL, Fernando HJS. Physical model of diurnal heating in the vicinity of a two-dimensional ridge. $J$ Atmos Sci 1996; 53: 62-85.

[8] Hunt JCR, Fernando HJS, Princevac M. Unsteady thermally driven flows on gentle slopes. J Atmos Sci 2003; 60: 2169-82.

[9] Schumann U. Large-eddy simulation of the upslope boundary layer. Q J R Meteor Soc 1990; 116: 637-670.

[10] Reuten C, Steyn DG, Allen SE. Water-Tank studies of atmospheric boundary layer structure and air-pollution transport in upslope flow systems. J Geophys Res 2007; 112: D11114.

[11] Mitsumoto S. A laboratory experiment on the slope wind. J Meteor Soc Japan 1989; 67: 565-74.

[12] Steyn DG. Scaling the vertical structure of sea breezes. BoundLayer Meteor 1998; 86: 505-24.

[13] Reuten C. "Scaling and kinematics of daytime slope flow systems," The University of British Columbia, Vancouver, Canada, 2006.

[14] Patel VC, Head MR. Some observations on skin friction and velocity profiles in fully developed pipe and channel flows. J Fluid Mech 1969; 38: 181-201.

[15] Stull RB. An introduction to boundary layer meteorology; Kluwer Academic Publishers: Dordrecht, The Netherlands 1988.

[16] Deardorff JW, Willis GE. Turbulence within a baroclinic laboratory mixed layer above a sloping surface. J Atmos Sci 1987; 44: $772-8$. 